\title{
Entada rheedii seeds thioamides, phenolics, and saponins and its antiulcerogenic and antimicrobial activities
}

\author{
Mona M. Okba ${ }^{*}$, Sally A.W. El Awdan², Miriam F. Yousif ${ }^{1,3}$, Kadriya S. El Deeb ${ }^{1}$, Fathy M. Soliman ${ }^{1}$ \\ ${ }^{1}$ Pharmacognosy Department, Faculty of Pharmacy, Cairo University, Kasr-El-Ainy St., Cairo 11562, Egypt. \\ ${ }^{2}$ Pharmacology Department, National Research Center, El-Tahrir St., Dokki, Cairo 12622, Egypt. \\ ${ }^{3}$ Department of Pharmacognosy and Medicinal Plants, Faculty of Pharmaceutical Sciences and Pharmaceutical Industries, Future University, Al Tagamoa Al \\ Khames, 11528, New Cairo, Egypt.
}

\begin{tabular}{|c|c|}
\hline ARTICLE INFO & ABSTRACT \\
\hline $\begin{array}{l}\text { Article history: } \\
\text { Received on: } 06 / 12 / 2017 \\
\text { Accepted on: } 16 / 02 / 2018 \\
\text { Available online: } 30 / 05 / 2018\end{array}$ & $\begin{array}{l}\text { Entada rheedii (Fabaceae) seeds are used against diarrhea and stomach aches. This study aims at scientifically validate } \\
\text { its ethnomedicinal uses. Seeds ethanol (70\%) extract (EE) was prepared by percolation. Antibacterial and antiviral } \\
\text { activities of EE and isolated compounds were determined using agar well diffusion and MTT assays, respectively. } \\
\text { Anti-ulcerogenic activity was evaluated using ethanol-induced ulcer model. Four phenolics: protocatechuic acid } \mathbf{C 1} \text {, } \\
\text { protocatechuic acid methyl ester C2, 1,3,4-trihydroxybenzene glucoside C3, phaseoloidin C4, three thioamides: }\end{array}$ \\
\hline $\begin{array}{l}\text { Key words: } \\
\text { Entada rheedii, rheedeioside } \\
\text { A, entadamide A, } \\
\text { phaseoloidin, gastric ulcer, } \\
\text { antibacterial. }\end{array}$ & $\begin{array}{l}\text { entadamide A C5, entadamide A- } \beta \text {-d-glucopyranoside C6, entadamide C C7, and two saponins: rheedeioside A C8 } \\
\text { and rheedeioside B C9 were isolated from EE. EE, C4, C5, and } \mathbf{C 8} \text { evidenced significant }(p<0.05) \text { antiulcerogenic } \\
\text { activity. Strong antibacterial activity was reported for EE, C1, and } \mathbf{C 7} \text {. C4 exhibited moderate ( } 35 \% \text { inhibition) } \\
\text { antiviral activity. This study provides scientific validation of the seed ethnomedicinal use in treating gastric ailments } \\
\text { and as antimicrobial. }\end{array}$ \\
\hline
\end{tabular}

\section{INTRODUCTION}

Entada rheedii Spreng. (Fabaceae) forests are found in tropical Asia; East Africa, Australia, Indian Ocean islands (Langran et al., 2009). It is widely distributed in tropical and subtropical countries bordering the Indian ocean. Its synonyms are E. pursaetha Candolle; E. pursaetha subsp. sinohimalensis. Long; E. pursaetha var. sinohimalensis (Grierson \& D. G), E. scandens (L) Benth. (Joshi, 2000). Most of its traditional uses were previously scientifically proved e.g. anti-inflammatory (Bhogireddy et al., 2015), wound healing (Vidya et al., 2012) and antidiarrheal activities (Dey et al., 2013). The plant folklore use in stomach problems (Bako et al., 2005; Uddin, 2006; Dey et al., 2013) and its use as antimicrobial (Dash and Padhy, 2006) (Vidya et al., 2012; Nzowa et al., 2013) is not yet fully investigated. This study investigates $E$. rheedii seeds potential in the treatment

"Corresponding Author

Mona M. Okba, Pharmacognosy Department, Faculty of Pharmacy, Cairo University, Kasr El-Ainy, 11562, Cairo, Egypt.

E-mail:mona.morad@pharma.cu.edu.eg of stomach ulcers especially that high levels of glutamic acid, a powerful antiulcerogenic amino acid, was reported before in the plant (Okba et al., 2013). Special emphasis was made on the evaluation of its antibacterial and antiviral potential. In addition, the scarcity of reports concerning the bioactive phytochemicals of the seeds of the cited plant encouraged the authors towards their isolation, identification, and assessment of their bioactivities.

\section{MATERIAL AND METHODS}

\section{Plant material}

E. rheedii Spreng. Seeds were purchased from the Experimental Station of Medicinal Plants, Pharmacognosy Department, Faculty of Pharmacy, Cairo University, Giza. Dr. Mohammed El-Gibali senior botanist confirmed its identity. The plant name has been checked with the International Plant Names Index (IPNI, 2004). Voucher samples (herbarium no. 14.4.2013.1) were deposited at the Museum of the Pharmacognosy Department, Faculty of Pharmacy, Cairo University. 


\section{Seeds extraction and fractionation}

Powdered seeds $(3 \mathrm{~kg})$ were defatted with $n$-hexane. Defatted mark was extracted using ethanol (70\%) by percolation at room temperature for $48 \mathrm{~h}$ yielding the ethanol extract (EE). EE (300 gm) was suspended in distilled water and successively fractionated using methylene chloride, and ethyl acetate. The EE fractions; methylene chloride (EE.MC), ethyl acetate (EE.EA), and remaining aqueous (EE.AQ), were used for isolation of the major compounds and biological evaluation.

\section{Isolation of the major constituents}

\section{General}

Stationary phases: Thin layer chromatography (TLC) was carried out on precoated silica gel F254 plates, $(20 \times 20 \mathrm{~cm}$, SigmaAldrich Chemicals-Germany). Meanwhile, various stationary phases were used for column chromatography (Cioffi et al., 2006) viz., nonion exchange resin (Diaion HP20 Sigma-Aldrich, St. Louis, MO, USA), Sephadex LH 20 (Pharmacia Fine Chemicals AB Uppsala, Sweden), silica gel 60 H (70-230 mesh, Fluka, Sigma-Aldrich chemicals-Germany) and Silica gel RP-18 (70-230 mesh, Fluka, Sigma-Aldrich chemicals-Germany) for medium pressure liquid chromatography (MPLC). Silica gel $\mathrm{H}$ was used for vacuum liquid chromatography (VLC). Solvents (of analytical grades) and authentic reference samples used were all purchased from E-Merck (Darmstadt, Germany). Solvent systems and spray reagents: S1: methylene chloride: methanol (9.1:0.9 v/v), S2: methylene chloride: methanol: formic acid (8.5:1.5:4 drops v/v/v), S3: methylene chloride: methanol: formic acid (6:4:20 drops v/v/v), S4: n-Butanol: acetic acid: water: methanol (4:1:1:0.5 v/v/v/v), S5: methylene chloride: methanol (7:3 $\mathrm{v} / \mathrm{v}$ ) were used. Spray reagents used were p-anisaldehyde-sulfuric acid, ferric chloride and iodine vapors (Smith, 1960; Stahl, 1969). Equipment and Apparatus: Chromatographic jars and glass columns of different dimensions were used for TLC and CC, respectively. UV lamp ( $\lambda_{\max }=254$ and $330 \mathrm{~nm}$, Shimadzu), a product of Hanovia lamps, was used for spot visualization on the chromatograms and a UV/Visible spectrophotometer, Shimadzu UV-1650 PC for recording UV spectra. Varian Mercury NMR-spectrometers (Japan) were used: $600 \mathrm{MHz}$ for recording ${ }^{1} \mathrm{H}-\mathrm{NMR}$ and $125 \mathrm{MHz}$ for ${ }^{13} \mathrm{C}-\mathrm{NMR}$; spectra were recorded in DMSO or CD3OD using TMS as internal standard and chemical shift values expressed in $\delta \mathrm{ppm}$. The mass of the compounds was detected using ESI-MS (electrospray ionization mass spectrometry) in negative mode.

\section{Phenolics isolation}

Part of the EE.EA ( $870 \mathrm{mg}$; TLC: 2 major spots, $\mathrm{R}_{\mathrm{f}} 0.62$ $\mathrm{S}_{1} \& 0.44 \mathrm{~S}_{1}$, both gave dull brown color under UV $254 \mathrm{~nm} \&$ bluish black color with ferric chloride) was chromatographed on Sephadex LH 20 and silica gel $60 \mathrm{H}$ columns resulted in isolation of $\mathbf{C} 1\left[\mathrm{R}_{\mathrm{f}}=0.62 \mathrm{~S}_{1}\right]$ and $\mathbf{C} 2\left[\mathrm{R}_{\mathrm{f}}=0.44 \mathrm{~S}_{1}\right]$.

\section{Thioamides isolation}

From EE.MC (2.5 g; TLC: 3 major spots, $\mathrm{R}_{\mathrm{f}} 0.58 \mathrm{~S}_{1}, 0.35$ $\mathrm{S}_{1}$ and $0.16 \mathrm{~S}_{5}$, the first two gave a dull brown color under short U.V. $254 \mathrm{~nm}$ while the third gave green color with $p$-anisaldehyde/ sulphuric) was enriched with fraction A from EE.EA (stated in 2.3.3.2 section) was fractionated on several silica gel $60 \mathrm{H}$ column resulted in isolation of $\mathbf{C 5}\left[\mathrm{R}_{\mathrm{f}}=0.58 \mathrm{~S}_{1} \& 0.82 \mathrm{~S}_{2}\right], \mathbf{C} 7\left[\mathrm{R}_{\mathrm{f}}=0.35\right.$ $\left.\mathrm{S}_{1}\right]$ and $\mathbf{C 3}\left[\mathrm{R}_{\mathrm{f}}=0.16 \mathrm{~S}_{5}\right]$.

From EE.EA (4.5 g; TLC: 2 major spots, $\mathrm{R}_{\mathrm{f}} 0.82 \mathrm{~S}_{2}$ $\left(=0.58 \mathrm{~S}_{1}\right) \& 0.25 \mathrm{~S}_{2}$, both gave a dull brown color under short U.V. $254 \mathrm{~nm}$, brown with iodine vapors and faint yellow and faint green colors after spraying with $p$-anisaldehyde/sulphuric reagent, respectively) was fractionated on Sephadex LH 20 column afforded two collective fractions A and B. Fraction A was added to methanol fractions of EE.MC and fractionated as mentioned before (section 2.3.3.1). Fraction B was purified over silica gel 60 $\mathrm{H}$ yielding $\mathbf{C 6}\left[\mathrm{R}_{\mathrm{f}}=0.25 \mathrm{~S}_{2}\right]$.

\section{Saponins isolation}

EE.AQ (150 g; TLC: 3 spots, $\mathrm{R}_{\mathrm{f}} 0.66 \mathrm{~S}_{4}, 0.28 \mathrm{~S}_{3}$ and $0.21 \mathrm{~S}_{3}$, the first gave green, greyish brown \& greyish brown color with $p$-anisaldehyde/sulphuric acid reagent respectively) were chromatographed over non-ion exchange resin (diaion). Two collective fractions I \& II were obtained. Fraction I was refractionated over a VLC, sephadex LH-20 followed by repeated $\mathrm{CC}$ using silica gel $60 \mathrm{H}$ columns to yield $\mathbf{C} 4\left[\mathrm{R}_{\mathrm{f}}=0.66 \mathrm{~S}_{4} \& 0.17\right.$ $\mathrm{S}_{2}$ ]. Fraction II was rechromatographed on a silica gel $60 \mathrm{H}$. The eluted fractions showed the presence of $\mathbf{C 4}$ and another spot $\left[\mathrm{R}_{\mathrm{f}}=\right.$ $0.28 \mathrm{~S}_{3}$ and $\left.0.21 \mathrm{~S}_{3}\right]$. They were rechromatographed on a sephadex LH 20 column affording two main sub-fractions, the first revealed on TLC a minor spot $\left[\mathrm{R}_{\mathrm{f}}=0.28 \mathrm{~S}_{3}\right]$ of $\mathbf{C 9}$; while the second upon purification on MPLC afforded $\mathbf{C 8}\left[\mathrm{R}_{\mathrm{f}}=0.21 \mathrm{~S}_{3}\right]$.

\section{Biological evaluation}

Done in accordance with ethical procedures and policies approved by the Animal Care and Committee of National Research Center, Dokki, Cairo.

$L D_{50}$

It was estimated using the acute toxic class method as per OECD guideline (Organization for Economic Co-operation and Development, Guideline-423, adopted on $17^{\text {th }}$ December 2001). After that, a dose-response curve was conducted for median effective dose determination.

\section{Antiulcerogenic activity}

Ethanol-induced ulcer model was adopted. Lesions were examined under an illuminated magnifier (Amaral et al., 2013).

\section{Antibacterial activity}

The agar well diffusion assay method as described by (Holder and Boyce, 1994) was adopted. Ten pathogenic bacteria were used Mycobacterium africanum, M. bovis, M. caprae, M. microti, M. orygis, Staphylococcus aureus, Streptococcus pneumoniae, Pseudomonas aeruginosa, Escherichia coli, Salmonella typhimurium (RCMB 0023645, 0023642, 00236940, 00236948, 00236941, 000106, 010010, 010043, 010052, 010072, respectively). The tested bacteria were subcultured on nutrient agar medium (Oxoid laboratories, UK). Gentamicin (Misr Company for Pharmaceutical Industry, Mataria, Cairo, Egypt) was used as a positive control. The diameter of inhibition zone was measured and the experiment was done in triplicate (Agwa et al., 2000). The minimum inhibitory concentration (MIC) and minimum bactericidal concentration were estimated according to 
(Doughari, 2006).

\section{Antiviral activity}

VERO cells (kidney epithelial cells of African green monkey) incubated into culture bottle were checked using the inverted microscope for its proper physical conditions. Healthy cells propagation, determination of extract cytotoxicity and MTT assay protocol were done according to (Gong, 2013).

\section{RESULTS AND DISCUSSION}

Chromatographic fractionation of the EE.ME, EE.EA and EE.AQ allowed the isolation of nine compounds. They were identified through their physicochemical characters, co-TLC comparison to authentic reference samples, spectroscopic analysis and through previously published data.

Compound C1: $29 \mathrm{mg}$, colorless needles. EI/MS at $\mathrm{m} / \mathrm{z}$ $153.12(\mathrm{M}-\mathrm{H})^{-}$. Its ${ }^{1} \mathrm{H}-\mathrm{NMR}$ spectral data showed signals at 7.5 $(1 \mathrm{H}, \mathrm{s}, \mathrm{H}-2), 6.8(1 \mathrm{H}, \mathrm{d}, \mathrm{J}=8.1 \mathrm{~Hz}, \mathrm{H}-5)$ and $7.45(1 \mathrm{H}, \mathrm{J}=8.1$ $\mathrm{Hz}, \mathrm{H}-6)$. Its ${ }^{13} \mathrm{C}-\mathrm{NMR}$ spectral data showed the following peaks: 124.1 (C1), 117.6 (C2), 151.09 (C3), 145.44 (C4), 115.7 (C5), 122.7 (C6) and 170.9 (C7).

Compound C2: $104 \mathrm{mg}$, pale buff needles. EI/MS at $\mathrm{m} / \mathrm{z}$ $167.14(\mathrm{M}-\mathrm{H})^{-}$. Its ${ }^{1} \mathrm{H}-\mathrm{NMR}$ spectral data showed signals at: 7.48 $(1 \mathrm{H}, \mathrm{s}, \mathrm{H}-2), 6.78(1 \mathrm{H}, \mathrm{d}, \mathrm{J}=8 \mathrm{~Hz}, \mathrm{H}-5), 7.39(1 \mathrm{H}, \mathrm{d}, \mathrm{J}=2.1 \mathrm{~Hz}$, H-6) and 3.821 (3H, s, H-8). Its ${ }^{13} \mathrm{C}-\mathrm{NMR}$ spectral data showed theses peaks: $122.5(\mathrm{C} 1), 115.8(\mathrm{C} 2), 146.12(\mathrm{C} 3), 151.64(\mathrm{C} 4)$, 117.41 (C5), 123.6 (C6), 168.83 (C7) and 52.22 (C8).

Compound $\mathrm{C} 3$ : $18 \mathrm{mg}$, white needles. EI/MS at $\mathrm{m} / \mathrm{z}$ $287.13(\mathrm{M}-\mathrm{H})$. Its ${ }^{1} \mathrm{H}-\mathrm{NMR}$ spectral data showed signals at 7.05 $(1 \mathrm{H}, \mathrm{d}, \mathrm{J}=6 \mathrm{~Hz}, \mathrm{H}-6), 6.668(1 \mathrm{H}, \mathrm{d}, \mathrm{J}=1.6 \mathrm{~Hz}, \mathrm{H}-3)$ and 6.607 $(1 \mathrm{H}, \mathrm{dd}, \mathrm{H}-5)$. Its ${ }^{13} \mathrm{C}-\mathrm{NMR}$ spectral data showed the following peaks 145.3 (C1), 153.84 (C2), 119.11 (C3), 150.6 (C4), 118.6 (C5), 115.23 (C6), 104.7 (C1'), 75.07 (C2'), 77.9 (C3'), 71.49 (C4'), 78.13 (C5') and 62.65 (C6').

Compound C4: $197 \mathrm{mg}$, white needles. EI/MS at $\mathrm{m} / z$ $329.14(\mathrm{M}-\mathrm{H})^{-}$. Its ${ }^{1} \mathrm{H}-\mathrm{NMR}$ spectral data showed: $6.67(1 \mathrm{H}, \mathrm{d}$, $\mathrm{J}=2.7 \mathrm{~Hz}, \mathrm{H}-3), 6.63(1 \mathrm{H}, \mathrm{dd}, \mathrm{J}=8.7 \mathrm{~Hz}, 3 \mathrm{~Hz}, \mathrm{H}-5)$ and 7.045 $(1 \mathrm{H}, \mathrm{d}, \mathrm{J}=8.7 \mathrm{~Hz}, \mathrm{H}-6)$. Its ${ }^{13} \mathrm{C}-\mathrm{NMR}$ spectral data showed the following peaks 149.15 (C1), 126.3 (C2), 117.08 (C3), 152.5 (C4), 117.8 (C5), 114.25 (C6), 35.5 (C7), 175.2 (C8), 103.2 (C1'), 73.7 (C2'), 76.5 (C3'), 70.04 (C4'), 76.7 (C5') and 61.26 (C6').

Compound C5: $78 \mathrm{mg}$, light brown syrup. EI/MS at $\mathrm{m} / \mathrm{z}$ $160.21(\mathrm{M}-\mathrm{H})^{-}$. Its ${ }^{1} \mathrm{H}-\mathrm{NMR}$ spectral data showed signals at: 5.81 $(1 \mathrm{H}, \mathrm{d}, \mathrm{J}=14.7 \mathrm{~Hz}, \mathrm{H}-2), 7.534(1 \mathrm{H}, \mathrm{d}, \mathrm{J}=14.7 \mathrm{~Hz}, \mathrm{H}-3), 6.2(1 \mathrm{H}$, s, NH), $3.377(2 \mathrm{H}, \mathrm{J}=4.2 \mathrm{~Hz}, \mathrm{CH} 2$ attached to $\mathrm{NH}), 3.646(2 \mathrm{H}$, $\mathrm{t}, \mathrm{J}=5.4 \mathrm{~Hz}, \mathrm{CH} 2$ beside $\mathrm{OH})$ and $2.311(3 \mathrm{H}, \mathrm{s}, \mathrm{CH} 3$ attached to $\mathrm{S})$. Its ${ }^{13} \mathrm{C}$-NMR spectral data showed the following peaks 164.12 (C1), 116.38 (C2), 143.29 (C3), 42.7 (NHCH2), 61.45 (CH2OH) and 14.4 (SCH3).

Compound C6: $23 \mathrm{mg}$, light brown syrup. EI/MS at $\mathrm{m} / \mathrm{z}$ 322.36 (M-H)-. Its ${ }^{1} \mathrm{H}-\mathrm{NMR}$ spectral data showed: $5.86(1 \mathrm{H}, \mathrm{d}, \mathrm{J}=14.7$ $\mathrm{Hz}, \mathrm{H}-2), 7.58(1 \mathrm{H}, \mathrm{d}, \mathrm{J}=14.7 \mathrm{~Hz}, \mathrm{H}-3), 2.32\left(3 \mathrm{H}, \mathrm{s}, \mathrm{CH}_{3}\right.$ attached to S) and $4.29\left(1 \mathrm{H}, \mathrm{d}, \mathrm{J}=7.5 \mathrm{~Hz}\right.$, anomeric proton). Its ${ }^{13} \mathrm{C}$-NMR spectral data showed the following peaks 167.49 (C1), 116.63 (C2), 143.82 (C3), 40.7 (NHCH2), 69.84 (CH2OH), 14.4 (SCH3), 104.56 (C1'), 75.14 (C2'), 78.006 (C3'\&C5'), 71.605 (C4') and 62.708 (C6').

Compound C7: $14 \mathrm{mg}$, white amorphous powder. EI/MS at $m / z$ 176.21 (M-H)- Its ${ }^{1} \mathrm{H}-\mathrm{NMR}$ spectral data showed: 6.707
$(1 \mathrm{H}, \mathrm{s}, \mathrm{J}=15 \mathrm{~Hz}, \mathrm{H}-2), 7.616(1 \mathrm{H}, \mathrm{d}, \mathrm{J}=15 \mathrm{~Hz}, \mathrm{H}-3), 3.644(2 \mathrm{H}$, $\mathrm{t}, \mathrm{J}=5.4 \mathrm{~Hz}, \mathrm{CH} 2$ attached to $\mathrm{NH}), 3.4(2 \mathrm{H}, \mathrm{t}, \mathrm{J}=5.4 \mathrm{~Hz}, \mathrm{CH} 2$ beside $\mathrm{OH})$ and $2.743(3 \mathrm{H}, \mathrm{s}, \mathrm{CH} 3$ attached to $\mathrm{S})$. Its ${ }^{13} \mathrm{C}-\mathrm{NMR}$ spectral data showed the following peaks 165.268 (C1), 129.3 (C2), 147.48 (C3), 43.3 (NHCH2), $61.339(\mathrm{CH} 2 \mathrm{OH})$ and 39.894 ( $\mathrm{SCH} 3)$.

Compounds C8: $83 \mathrm{mg}$, transparent amorphous powder. Its ${ }^{13} \mathrm{C}$-NMR spectral data showed the following peaks $38.35(\mathrm{C} 1)$, 25.6 (C2), 88.27 (C3), 38.37 (C4), 54.77 (C5), 18.01 (C6), 36.30 (C7), 40.03 (C8), 46.68 (C9), 36.40 (C10), 23.08 (C11), 123.81 (C12), 143.22 (C13), 47.32 (C14), 67.00 (C15), 77.6 (C16), 47.44 (C17), 40.37 (C18), 46.41 (C19), 30.13 (C20), 32.97 (C21), 30.19 (C22), 27.50 (C23), 16.3 (C24), 15.30 (C25), 17.10 (C26), 19.70 (C27), 174.90 (C28), 32.886 (C29), 24.05 (C30), 103.6 (C1'), 55.13 (C2'), 79.56 (C3'), 73.21 (C4'), 75.74 (C5'), 67.806 (C6'), 102.7 (C1"), 73.44 (C2"), 76.44 (C3"), 69.29 (C4"), 64.05 (C5"), 101.8 (C1"'), 71.91 (C2"'), 81.61 (C3"”) 66.92 (C4"”), 76.33 (C5"”), 63.31 (C6"'), 106.2 (C1"'”), 74.79 (C2"'”), 76.74 (C3"'”), 69.55 (C4"”'), 65.89 (C5"'”), 92.34 (C1"'”'), 79.14 (C2"”"'), 81.95

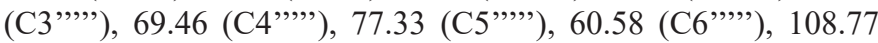
(C1"',"'), 77.58 (C2"',"'), 79.19 (C3"',"'), 73.39 (C4"',"'), 69.39

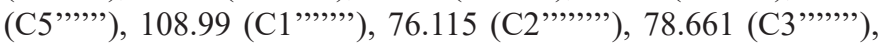
72.16 (C4"','”), 63.49 (C5',','), $20.65\left(\mathrm{NHCOCH}_{3}\right), 168.50$ $\left(\mathrm{NHCOCH}_{3}\right), 19.97\left(\mathrm{OCOCH}_{3}\right), 170.20\left(\mathrm{OCOCH}_{3}\right)$.

Compounds C9: $23 \mathrm{mg}$ transparent amorphous powder. Its ${ }^{13} \mathrm{C}$-NMR spectral data showed the following peaks $38.43(\mathrm{C} 1)$, 25.91 (C2), 88.39 (C3), 38.45 (C4), 54.65 (C5), 18.08 (C6), 36.26 (C7), 40.15 (C8), 46.55 (C9), 36.39 (C10), 23.16 (C11), 124.10 (C12), 143.20 (C13), 47.45 (C14), 67.19 (C15), 77.93 (C16), 47.63 (C17), 40.47 (C18), 46.39 (C19), 30.14 (C20), 33.04 (C21), 30.22 (C22), 27.69 (C23), 16.45 (C24), 15.32 (C25), 17.41 (C26), 19.73 (C27), 174.98 (C28), 32.89 (C29), 24.12 (C30), 105.00 (C1'), 57.25 (C2'), 82.4 (C3'), 75.01 (C4'), 76.91 (C5'), 70.03 (C6'), 105.8 (C1'), 76.25 (C2"), 78.5 (C3"), 72.68 (C4"), 66.09 (C5"), 103.8 (C1"”), 74.76 (C2"”), 84.70 (C3"”), 69.78 (C4"”), 79.98 (C5"”), 64.82 (C6"”), 111.5 (C1"”'), 79.8 (C2"'"), 81.23 (C3"”'), 74.36 (C4"”), 70.9 (C5"”), 93.9 (C1"'”'), 80.56 (C2"'”'), 83.39 (C3"'”'), 71.24 (C4"'”), 78.68 (C5"'”), 62.34 (C6"'”), 107.00 (C1"'”"'), 76.09 (C2"',"'), 77.60 (C3"',"'), 72.10 (C4"'"'), 68.03 (C5"'"'), 111.8 (C1"',"'), 79.24 (C2"'י"'"),

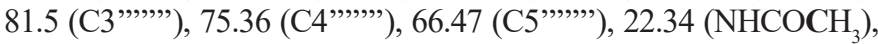
$169.97\left(\mathrm{NHCOCH}_{3}\right)$.

Compounds $\mathrm{C} 1$ and $\mathrm{C} 2{ }^{1} \mathrm{H}$ and ${ }^{13} \mathrm{C}-\mathrm{NMR}$ in agreement with the reported data of protocatechuic acid and protocatechuic acid methyl ester respectively (Kwak et al., 2009). Compounds $\mathrm{C} 3, \mathrm{C} 4, \mathrm{C} 5, \mathrm{C} 6$, and $\mathrm{C} 7{ }^{1} \mathrm{H}$ and ${ }^{13} \mathrm{C}-\mathrm{NMR}$ in agreement with the reported data of 1,3,4 trihydroxybenzene glucoside (Pan and Lundgren, 1995), phaseoloidin (Barua et al., 1988), entadamide A (Fumio et al., 1985), entadamide A- $\beta$-D-glucopyranoside (Dai et al., 1991), entadamide C (Ikegami et al., 1989) respectively. Compounds $\mathrm{C} 8$ and $\mathrm{C} 9{ }^{1} \mathrm{H},{ }^{13} \mathrm{C}-\mathrm{NMR}$, COSY, HSQC (Table 1) and $\mathrm{HMBC}$ in agreement with the reported data of rheedeioside $\mathrm{A}$ and rheedeioside B respectively (Sugimoto et al., 2011).

Structures of C1-9 are presented in Figure 1. Phaseoloidin, entadamide A- $\beta$-D-glucopyranoside, rheedeioside A and rheedeioside B were previously reported in E. rheedii seeds (Tapondjou et al., 2005; Sugimoto et al., 2011). However, this is the first report for the presence of protocatechuic acid, protocatechuic acid methyl ester, 1,3,4 trihydroxybenzene glucoside, entadamide $\mathrm{A}$ and entadamide $\mathrm{C}$ in this seeds. 
<smiles>O=C(O)c1ccc(O)c(O)c1</smiles>

C1:

C2:<smiles>[R6]CCNC(=O)/C=C\SC</smiles>

C5: C6:

$$
\begin{aligned}
& \mathrm{R}=\mathrm{H} \\
& \mathrm{R}=\text { Glucose }
\end{aligned}
$$<smiles>[2H]c1cc(O)ccc1O[C@@H]1O[C@H](CO)[C@@H]2O[C@H]1[C@H]2O</smiles>

C3: $\quad \mathrm{R}=\mathrm{OH}$

C4: $\quad \mathrm{R}=\mathrm{CH}_{2} \mathrm{COOH}$<smiles>CS(=O)C=CC(=O)NCCO</smiles>

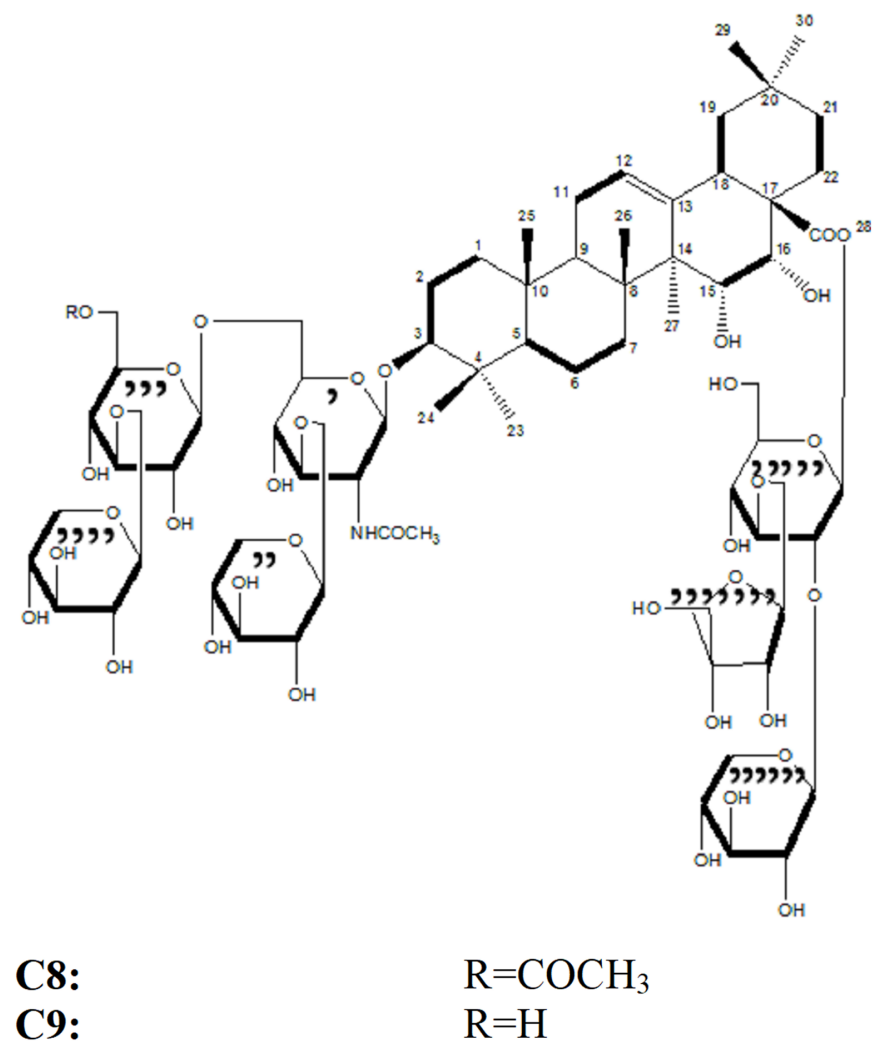

Fig. 1: Structures of the isolated compounds C1-9. 
Table 1: HSQC Spectroscopic correlation between anomeric carbons and protons of Compounds $\mathbf{C 8}$ and $\mathbf{C 9}$.

\begin{tabular}{ccccc}
\hline & \multicolumn{2}{c}{ C8 } & \multicolumn{2}{c}{ C9 } \\
\cline { 2 - 5 } & Anomeric c & Anomeric H & Anomeric c & Anomeric H \\
\hline C3 sugars & & & & \\
, & 103.606 & 4.51 & 105 & 4.62 \\
,, & 102.792 & 4.44 & 105.8 & 4.41 \\
,$"$, & 101.824 & 4.36 & 103.8 & 4.59 \\
,,$"$ & 106.228 & 4.23 & 111.5 & 4.95 \\
C28 sugars & & & & \\
,"," & 92.34 & 5.38 & 93.9 & 5.45 \\
,",", & 108.77 & 5.27 & 107 & 4.43 \\
,",", & 108.99 & 4.77 & 111.8 & 5.3 \\
\hline
\end{tabular}

Previous investigations have shown that several Entada species contain saponins in considerable amounts. Several workers isolated saponins from different Entada species (Tapondjou et al., 2005; Cioffi et al., 2006; Sugimoto et al., 2011). Many Entada pharmacological activities were related to its saponins (Liu et al., 1972; Yasuraoka et al., 1977; Dai et al., 1991; Cioffi et al., 2006). The lack of information concerning the relationship between E. rheedii biophytochemicals and pharmacological activities encouraged the authors to study the relation between each studied activity and the isolated thioamides, phenolics, and saponins.

No mortality was observed after one-day administration of EE limit dose up to $4 \mathrm{~g} / \mathrm{kg}$ b. wt. The experiment was repeated using 3 additional animals at the same dose and no mortality was observed again. In accordance with the Acute Toxic Class Method reported in OECD guideline\# 423. The EE is considered to be GHS unclassified with $\mathbf{L D}_{\mathbf{5 0}}$ cut-off $>5000 \mathrm{mg} / \mathrm{kg}$. Antiulcerogenic activity (Table 2$)$. Its activity exceeded that of omeprazole $(20 \mathrm{mg} /$ $\mathrm{kg}$ ) and approached that of sucralfate $(400 \mathrm{mg} / \mathrm{kg})$ and ranitidine
(50 mg/kg). EE reduced ulcers number and severity by 95.24 and $97.98 \%$ respectively. Thus $E$. rheedii seeds antiulcerogenic activity is more than E. phaseoloides seeds antiulceroginic activity reported before by (Ramakrishna et al., 2008). All tested $\mathrm{EE}$ fractions and isolated compounds (C4, C5 \& C8 major isolated phenolic, thioamide, and saponin respectively) have significant ( $p$ $<0.05)$ antiulcerogenic activity. EE.EA was the most powerful in the reduction of both ulcer number and severity followed by EE.MC. It reduced ulcer number and severity by 92.86 and $97.25 \%$ respectively. It deserves to mention that EE.EA is the fraction from which thioamides and phenolics were isolates. Rheedeioside A C8 was more potent antiulcerogenic than phasoledin $\mathbf{C 4}$ and entadamide A C5. It reduced ulcer number and severity by 92.86 and $96.33 \%$ respectively. Thus, the antiulcerogenic activity was not attributed to specific biophytochemical class. The powerful antiulcerogenic activity of $E$. rheedii seeds might be also attributed to high levels of glutamic acid (Okba et al., 2013). Glutamic acid is known in the treatment of ulcers (Blitz et al., 1963; Repetto and Llesuy, 2002).

EE $(1 \mathrm{mg} / \mathrm{ml})$ showed moderate to strong antibacterial activity against 8 strains of the 10 tested pathogenic bacteria except for M. orygis and P. aeruginosa which were resistant (Table $3)$. EE has high antibacterial activity relative to standard reference gentamicin (SRG). It exhibited its antibacterial activity through bactericidal action against all sensitive strains. The activity of EE against $S$. typhi reached $96 \%$ of SRG. The seeds can be used in urinary tract infections due to the potent bactericidal activity of EE against E. coli and $S$. aureus as same as SRG potency. This potent antimicrobial activity is possibly due to the seed content of various classes of phytochemicals. Saponins (Pavithra et al., 2010), phenolics (Okoro et al., 2010), and sulfur compounds (Kim et al., 2006) are among classes of well documented antibacterial activity.

Table 2: Effect of E. rheedii seed on gastric ulcer number and severity.

\begin{tabular}{|c|c|c|c|c|c|}
\hline \multirow{2}{*}{ Treatment } & \multirow{2}{*}{ Dose $(\mathrm{mg} / \mathbf{k g})$} & \multicolumn{2}{|c|}{ Ulcer number } & \multicolumn{2}{|c|}{ Ulcer severity } \\
\hline & & Mean \pm SE & $\%$ Reduction & Mean \pm SE & $\%$ Reduction \\
\hline Control & & 0 & & 0 & \\
\hline 99.5\% Ethanol & & $8.4 \pm 0.005^{*}$ & & $21.8 \pm 0.124^{*}$ & \\
\hline $\mathrm{EE}$ & 200 & $0.40 \pm 0.244 * @$ & 95.24 & $0.44 \pm 0.040 * @$ & 97.98 \\
\hline $\mathrm{EE}$ & 100 & $0.66 \pm 0.333 * @$ & 92.14 & $0.97 \pm 0.214 * @$ & 95.52 \\
\hline EE.MC & & $0.8 \pm 0.065 * @$ & 90.48 & $2.6 \pm 0.135^{*} @$ & 88.1 \\
\hline EE.EA & & $0.6 \pm 0.002 * @$ & 92.86 & $0.6 \pm 0.001 * @$ & 97.25 \\
\hline $\mathrm{C} 5$ & & 0.8×0.001*@ & 90.48 & $1.4 \pm 0.015^{*} @$ & 93.58 \\
\hline $\mathrm{C} 4$ & & $0.8 \pm 0.003 * @$ & 90.48 & $5.6 \pm 0.08 * @$ & 74.32 \\
\hline $\mathrm{C} 8$ & & $0.6 \pm 0.011 * @$ & 92.86 & $0.8 \pm 0.04 * @$ & 96.33 \\
\hline Omeprazole & 20 & $1 \pm 0.003 * @$ & 88.1 & $2 \pm 0.021 * @$ & 90.83 \\
\hline Sucraflate & 400 & $0.4 \pm 0.004 * @$ & 95.24 & $0.4 \pm 0.002 * @$ & 98.2 \\
\hline Ranitidine & 50 & $0.33 \pm 0.004 * @$ & 96.1 & $0.33 \pm 0.001 * @$ & 98.5 \\
\hline
\end{tabular}

Each value represents the mean of 6 rats \pm SE of the mean. Statistical analysis was carried out using Kruskal-Wallis non parametric one way ANOVA. EE: ethanol 70\% extract. Fractions of ethanol 70\% extract: methylene chloride (EE.MC), and ethyl acetate (EE.EA). *Statistically significant from the normal p $<0.05$, @Statistically significant from the control ulcer $\mathrm{p}<0.05$. phaseoloidin $\mathrm{C} 4$, entadamide A C5 and rheedeioside A C8.

Fractionation of EE showed that both EE.EA and EE.MC had potent antibacterial activity. EE.AQ had no activity against all sensitive strains. It is worth to note that the potent fractions (EE. EA and EE.MC) are the two fractions from which thioamides and 
phenolics were isolated. It is obvious that fractionation of the EE to EE.EA increases the antibacterial activity against five strains by $4-16 \%$ of SRG and decreased the activity against $S$. aureus and $M$. microti by $4-12 \%$ of SRG. EE.EA exerted significantly higher activity than EE.MC against all sensitive strains except M. africanum. The absence of activity in EE.AQ, the fraction from which saponins C8 \& C9 were isolated, indicates that the antibacterial activity is not due to the seed saponins content. On the other hand, the potent activity of EE.EA and EE.MC indicated that the antimicrobial activity is related to thioamides and phenolics. This was also confirmed when testing the antibacterial potency of the isolated saponins $\mathrm{C} 8$ and $\mathrm{C} 9 . \mathrm{C} 8$ exhibits moderate to weak activity against 3 strains only while C9 was completely inactive.

Table 3: Antibacterial activity of E. rheedii seeds.

\begin{tabular}{|c|c|c|c|c|c|c|c|c|c|c|c|c|c|c|c|}
\hline & & & \multicolumn{4}{|c|}{ Extract and fractions } & \multicolumn{3}{|c|}{ Phenolics } & \multicolumn{3}{|c|}{ Thioamides } & \multicolumn{2}{|c|}{ Saponins } & \multirow{2}{*}{ RSG } \\
\hline & & & $\mathbf{E E}$ & EE.MC & EE.EA & EE.AQ & C1 & $\mathrm{C} 2$ & $\mathrm{C} 4$ & C5 & C6 & $\mathrm{C} 7$ & $\mathrm{C8}$ & C9 & \\
\hline \multirow{16}{*}{ Mycobacteria } & \multirow{4}{*}{ M.a. } & $\mathrm{D}(\mathrm{mm})^{*}$ & 22 & 24 & 22 & - & 23 & 20 & 18 & 18 & 14 & 20 & - & - & 24 \\
\hline & & $\mathrm{D}(\%)^{* *}$ & 92 & 100 & 92 & & 96 & 83 & 75 & 75 & 58 & 83 & & & 100 \\
\hline & & MIC & 0.24 & 0.12 & 0.49 & - & 0.24 & 3.9 & 15.63 & 7.81 & 31.25 & 0.98 & - & - & 0.06 \\
\hline & & MBC & 0.24 & 0.49 & 0.49 & - & 0.24 & 3.9 & 15.63 & 31.25 & 62.5 & 0.98 & - & - & 0.06 \\
\hline & \multirow{4}{*}{ M.b. } & $\mathrm{D}(\mathrm{mm})^{*}$ & 20 & 21 & 23 & - & 24 & 21 & 19 & 21 & 16 & 22 & - & - & 19 \\
\hline & & $\mathrm{D}(\%)^{* *}$ & 105 & 111 & 121 & & 126 & 111 & 100 & 111 & 84 & 116 & & & 100 \\
\hline & & MIC & 3.9 & 1.95 & 0.24 & - & 0.12 & 1.95 & 7.81 & 1.95 & 31.25 & 0.49 & - & - & 1.95 \\
\hline & & $\mathrm{MBC}$ & 3.9 & 7.81 & 0.24 & - & 0.12 & 1.95 & 7.81 & 3.9 & 62.5 & 0.49 & - & - & 1.95 \\
\hline & \multirow{4}{*}{ M.c. } & $\mathrm{D}(\mathrm{mm})^{*}$ & 23 & 23 & 24 & - & 25 & 22 & 21 & 21 & 18 & 22 & - & - & 24 \\
\hline & & $\mathrm{D}(\%)^{* *}$ & 96 & 96 & 100 & & 104 & 92 & 88 & 88 & 75 & 92 & & & 100 \\
\hline & & MIC & 0.24 & 0.24 & 0.24 & - & 0.12 & 0.49 & 0.98 & 0.98 & 15.63 & 0.24 & - & - & 0.06 \\
\hline & & MBC & 0.24 & 0.98 & 0.24 & - & 0.12 & 0.49 & 0.98 & 3.9 & 31.25 & 0.24 & - & - & 0.06 \\
\hline & \multirow{4}{*}{ M.m. } & $\mathrm{D}(\mathrm{mm})^{*}$ & 24 & 20 & 21 & - & 22 & 19 & 22 & 19 & 16 & 22 & - & - & 24 \\
\hline & & $\mathrm{D}(\%)^{* *}$ & 100 & 83 & 88 & & 92 & 79 & 92 & 79 & 67 & 92 & & & 100 \\
\hline & & MIC & 0.12 & 1.95 & 1.95 & - & 0.98 & 3.9 & 0.49 & 7.81 & 31.25 & 0.49 & - & - & 0.06 \\
\hline & & $\mathrm{MBC}$ & 0.12 & 3.9 & 1.95 & - & 0.98 & 3.9 & 0.49 & 15.63 & 62.5 & 0.49 & - & - & 0.06 \\
\hline \multirow{8}{*}{ Gram +} & \multirow{4}{*}{ S.a. } & $\mathrm{D}(\mathrm{mm})^{*}$ & 23 & 20 & 22 & - & 22 & 20 & nd & 16 & 16 & 19 & 13 & - & 25 \\
\hline & & $\mathrm{D}(\%)^{* *}$ & 92 & 80 & 88 & & 88 & 80 & & 64 & 64 & 76 & 52 & & 100 \\
\hline & & MIC & 0.49 & 3.9 & 0.49 & - & 0.24 & 1.95 & $\mathrm{Nd}$ & 31.25 & 31.25 & 3.9 & 500 & - & 0.03 \\
\hline & & MBC & 0.49 & 3.9 & 0.49 & - & 0.24 & 1.95 & $\mathrm{Nd}$ & 62.5 & 62.5 & 3.9 & 1000 & - & 0.03 \\
\hline & \multirow{4}{*}{ S.p. } & $\mathrm{D}(\mathrm{mm})^{*}$ & 18 & 21 & 22 & - & 24 & 17 & nd & 19 & 13 & 21 & 15 & - & 20 \\
\hline & & $\mathrm{D}(\%)^{* *}$ & 90 & 105 & 110 & & 120 & 85 & & 95 & 65 & 105 & 75 & & 100 \\
\hline & & MIC & 7.81 & 0.98 & 0.98 & - & 0.12 & 31.25 & $\mathrm{Nd}$ & 3.9 & 125 & 0.98 & 125 & - & 1.95 \\
\hline & & MBC & 7.81 & 7.8 & 0.98 & - & 0.12 & 62.5 & $\mathrm{Nd}$ & 31.25 & 500 & 0.98 & 500 & - & 1.95 \\
\hline \multirow{8}{*}{ Gram - } & \multirow{4}{*}{ E.c. } & $\mathrm{D}(\mathrm{mm})^{*}$ & 24 & - & 23 & - & 24 & 15 & nd & 18 & 15 & 15 & - & - & 24 \\
\hline & & $\mathrm{D}(\%)^{* *}$ & 100 & & 96 & & 100 & 63 & & 75 & 63 & 63 & & & 100 \\
\hline & & MIC & 0.12 & - & 0.24 & - & 0.06 & 31.25 & $\mathrm{Nd}$ & 15.63 & 62.5 & 62.5 & - & - & 0.06 \\
\hline & & MBC & 0.12 & - & 0.24 & - & 0.06 & 62.5 & $\mathrm{Nd}$ & 62.5 & 125 & 62.5 & - & - & 0.06 \\
\hline & \multirow{4}{*}{ S.t. } & $\mathrm{D}(\mathrm{mm})^{*}$ & 25 & - & 24 & - & 22 & 20 & nd & 19 & 15 & 16 & 12 & - & 26 \\
\hline & & $\mathrm{D}(\%)^{* *}$ & 96 & & 92 & & 85 & 77 & & 73 & 58 & 62 & 46 & & 100 \\
\hline & & MIC & 0.12 & - & 0.12 & - & 0.24 & 1.95 & $\mathrm{Nd}$ & 3.9 & 62.5 & 62.5 & 500 & - & 0.03 \\
\hline & & $\mathrm{MBC}$ & 0.12 & - & 0.12 & - & 0.24 & 7.81 & $\mathrm{Nd}$ & 3.9 & 62.5 & 62.5 & 500 & - & 0.03 \\
\hline
\end{tabular}

*Mean zone of inhibition in $\mathrm{mm}$ beyond good diameter $(6 \mathrm{~mm})$ produced on a range clinically pathogenic bacteria using $(1 \mathrm{mg} / \mathrm{ml})$ concentration of tested samples. **\% inhibition relative to gentamicin. MICs and MBCs $(\mu \mathrm{g} / \mathrm{ml})$. EE: $70 \%$ extract. Fractions of ethanol $70 \%$ extract: methylene chloride (EE.MC), ethyl acetate (EE. EA), n-butanol (EE.BU) and aqueous (EE.AQ) M. a., Mycobacterium africanum, M. b., Mycobacterium bovis, M.c., Mycobacterium caprae, M.m., Mycobacterium microti, S.a., Staphylococcus aureus, S.p., Streptococcus pneumoniae, E.c., Escherichia coli, S.t., Salmonella typhimurium. nd., not done. protocatechuic acid C1, protocatechuic acid methyl ester $\mathrm{C}$, phaseoloidin $\mathrm{C} 4$, entadamide A C5, entadamide A- $\beta$-D-glucopyranoside C6, entadamide C C7, rheedeioside A C8 and rheedeioside B C9. nd; not done. RSG; reference standard genatmycin.

Concerning phenolics: protocatechuic acid $\mathbf{C 1}$ is more active than its methyl ester $\mathbf{C 2}$. Its activity exceeds SRG against certain strains e.g. M. bovis (126\%), S. pneumonia (120\%) and $M$. caprae (104\%) of SRG. Phaseolidin C4 activity ranges from $75 \%$ to $100 \%$ of SRG on the sensitive strains. Concerning thioamides: entadamide C C7 was much more active than entadamide A C5 and its glucoside C6 against all sensitive gram-positive and mycobacterium bacteria, while entadamide A C5 was more active 
than other thioamides against sensitive gram-negative bacteria. Entadamide A C5 had a potent antibacterial activity than its corresponding glucoside $\mathbf{C 6}$ against all sensitive strains except $S$. aureus which is affected equally by both. Both saponins (C8 and C9) are inactive against all mycobacterium strains. Only rheedeioside A C8 showed a moderate activity (46\%, 52\%, and $75 \%$ of SRG) against S. typhimurium, S. aureus, and S. pneumonia respectively. Thus, it is concluded that the antibacterial activity is related to phenolics and thioamides not to the saponin content.

Studying MIC and MBC revealed that, EE.EA, Protocatechuic acid $\mathbf{C 1}$, phaseoledine $\mathbf{C 4}$ and entadamide $\mathrm{C} \mathbf{C 7}$ exhibited bactericidal action against all sensitive strains. EE.MC and rest of tested compounds showed bacteriostatic activity against some strains and bactericidal activity against others as shown in Table 3.

Table 4: Antiviral effect of E. rheedii seed against CoxB4 virus.

\begin{tabular}{cccccc}
\hline Test sample & $\begin{array}{c}\text { MNTC (mg/ } \\
\text { ml) [MNTC] }\end{array}$ & O.D & $\begin{array}{c}\text { Viabilityy } \\
\%\end{array}$ & $\begin{array}{c}\text { Cytotoxicity } \\
\%\end{array}$ & $\begin{array}{c}\text { Anti-viral } \\
\text { effect }\end{array}$ \\
\hline VERO cell line & & 0.113 & $100 \%$ & $0 \%$ & $0 \%$ \\
Virus control & & 0.035 & 31 & 69 & \\
EE & 0.1 & 0.057 & 50 & 50 & $19 \% *$ \\
C4 & 0.1 & 0.075 & 66 & 34 & $35 \% *$ \\
C5 & 0.1 & 0.042 & 37 & 63 & $6 \%$ \\
C6 & 0.1 & 0.057 & 40 & 50 & $19 \% *$ \\
C7 & 0.1 & 0.045 & 40 & 60 & $9 \%$ \\
C8 & 0.01 & 0.032 & 28 & 72 & $0 \%$ \\
C9 & 0.1 & 0.044 & 39 & 61 & $8 \%$ \\
\hline
\end{tabular}

EE: ethanol 70\% extract. MNTC, Maximum non-toxic concentration; O.D., Optical density; *average of six determinations. phaseoloidin $\mathrm{C} 4$, entadamide $\mathrm{A}$ $\mathrm{C} 5$, entadamide A- $\beta$-D-glucopyranoside C6, entadamide C $\mathrm{C} 7$, rheedeioside A $\mathrm{C} 8$ and rheedeioside B C9.

M. bovis was the most sensitive bacteria to E. rheedii seed tested fractions and isolates Table 3, its sensitivity ranged from $79 \%$ to $126 \%$ of SRG. M. bovis was inhibited by some samples with very low MICs ranging from $0.12,0.24,0.49 \mu \mathrm{g} / \mathrm{ml}$ by protocatechuic acid C1, EE.EA and entadamide C C7. These MICs are lower than that of SRG $(1.95 \mu \mathrm{g} / \mathrm{ml})$. M. bovis is the causative agent of tuberculosis in cattle, known as bovine TB, and can jump the species barrier and cause tuberculosis in human (Grange et al., 1996). The pronounced activities against M. bovis and $M$. caprae, which can cause tuberculosis also (Rodríguez et al., 2009), validate the seeds ethnomedicinal use in tuberculosis treatment (Vidya et al., 2012). All isolated compounds exert lower antityphoid activity (46-85\% of SRG) when compared to EE ( $96 \%$ of SRG) which indicated the necessity of synergism between different classes of biophytochemicals to exert such potent antityphoid activity. S. aureus and E. coli are among bacteria that cause gastroenteritis and diarrhea (Jarraud et al., 2001; Becker et al., 2003; Al-Gallas et al., 2007; Rodríguez et al., 2009). EE and isolated compounds exhibited potent activity against this species, this validates the seeds ethnomedicinal use in gastroenteritis and diarrhea.

EE has weak antiviral activity against Coxsackie B4 virus Table 4 . It reduced the virus activity by $19 \%$. This virus can trigger an autoimmune reaction resulting in the destruction of pancreatic insulin-producing beta cells causing diabetes mellitus (Ylipaasto et al., 2004). Phasoloidin C4 and entadamide A- $\beta$-Dglucopyranoside $\mathbf{C 6}$ were the most active antiviral constituents. They reduced the activity of Coxsackie B4 virus by 35 and $19 \%$ respectively. Phasoloidin $\mathbf{C 4}$ the major isolated phenolic is much more potent than EE while entadamide $\mathrm{A}-\beta$-D-glucopyranoside C6 has antiviral activity equal to that of EE. Subsequently, the antiviral activity is related mainly to the seed phenolics especially that the other tested thioamides and saponins have almost neglected activities ranged from $0-9 \%$.

This is the first report on antibacterial, antiviral and antiulcerogenic activities of $E$. rheedii seeds biophytochemicals.

\section{CONCLUSION}

E. rheedii seeds possess potent antiulcerogenic and antimicrobial activities. This explains its traditional use in the treatment of stomach aches and as an antimicrobial.

\section{ACKNOWLEDGMENT}

The Authors would like to thank Prof. Dr. Mohammed Farag and Assistant Prof. Dr. Ali El Halawany, Faculty of pharmacy Cairo University for their help in NMR analysis of the isolated compounds.

\section{FUNDING}

This research received no specific grant from any funding agency in the public, commercial or not-for-profit sector.

\section{REFERENCES}

Agwa A, Aly M, Bonaly R. Isolation and characterization of two Streptomyces species produced non polyenic antifungal agents. J. Union Arab Biol. 2000; 7:62-82.

Al-Gallas N, Bahri O, Bouratbeen A, Haasen AB, Aissa RB. Etiology of acute diarrhea in children and adults in Tunis, Tunisia, with emphasis on diarrheagenic Escherichia coli: prevalence, phenotyping, and molecular epidemiology. The American journal of tropical medicine and hygiene. 2007; 77:571-582.

Amaral GP, de Carvalho NR, Barcelos RP, Dobrachinski F, de Lima Portella R, da Silva MH, Lugokenski TH, Dias GRM, da Luz SCA, Boligon AA. Protective action of ethanolic extract of Rosmarinus officinalis L. in gastric ulcer prevention induced by ethanol in rats. Food and Chemical Toxicology. 2013; 55:48-55.

Bako S, Bakfur M, John I, Bala E. Ethnomedicinal and phytochemical profile of some savanna plant species in Nigeria International Journal of Botany. 2005; 1(2):147-150.

Barua AK, Chakrabarty M, Datta PK, Ray S. Phaseoloidin, a homogentisic acid glucoside from Entada phaseoloides. Phytochemistry. $1988 ; 27: 3259-3261$

Becker K, Friedrich AW, Lubritz G, Weilert M, Peters G, von Eiff C. Prevalence of genes encoding pyrogenic toxin superantigens and exfoliative toxins among strains of Staphylococcus aureus isolated from blood and nasal specimens. Journal of clinical microbiology. 2003; 41:1434-1439.

Bhogireddy N, Mathi P, Ambatipudi N, Talluri V, Bokka VR. In vitro anti-inflammatory and biofractionation of Entada pursaetha DC ethanol seed extract in LPS induced RAW 264.7 macrophage cells. Adv Biol Res. 2015; 9:109-116.

Blitz J, Smith JW, Gerard JR. Aloe vera gel in peptic ulcer therapy; Preliminary report. Journal AOA. 1963; 62: 731-735.

Cioffi G, Dal Piaz F, De Caprariis P, Sanogo R, Marzocco S, Autore G, De Tommasi N. Antiproliferative Triterpene Saponins from Entada africana. Journal of natural products. 2006; 69:1323-1329. 
Dai J, Kardono LB, Tsauri S, Padmawinata K, Pezzuto JM, Kinghorn AD. Phenylacetic acid derivatives and a thioamide glycoside fromEntada phaseoloides. Phytochemistry. 1991; 30:3749-3752.

Dash SK, Padhy S. Review on ethnomedicines for diarrhoea diseases from Orissa: prevalence versus culture. J Hum Ecol. 2006; 20:5964.

Dey S, Rahman M, Hira A, Ahmed A, Howlader MS, Khatun A, Siraj MA. Phytochemical screening and pharmacological activities of Entada scandens seeds. International Journal of Applied Research in Natural Products. 2013; 6:20-26.

Doughari J. Antimicrobial activity of Tamarindus indica Linn. Tropical Journal of Pharmaceutical Research. 2006; 5:597-603.

Fumio I, Isao S, Shigeru O, Nijsiri R, Isamu M. Entadamide A, a new sulfur-containing amide from Entada phaseoloides seeds. Chemical \& pharmaceutical bulletin. 1985; 33:5153-5154

Gong EY. 2013. Antiviral methods and protocols, Springer.

Grange JM, Yates MD, de Kantor IN, Organization WH. Guidelines for speciation within the Mycobacterium tuberculosis complex. 1996.

Holder I, Boyce S. Agar well diffusion assay testing of bacterial susceptibility to various antimicrobials in concentrations non-toxic for human cells in culture. Burns. 1994; 20:426-429.

Ikegami F, Sekine T, Duangteraprecha S, Matsushita N, Matsuda $\mathrm{N}$, Ruangrungsi N, Murakoshi I. Entadamide C, a sulphur-containing amide from Entada phaseoloides. Phytochemistry. 1989; 28:881-882.

IPNI (2004). The International Plant Names Index. Entada rheedei.

Jarraud S, Peyrat MA, Lim A, Tristan A, Bes M, Mougel C, Etienne J, Vandenesch F, Bonneville M, Lina G. egc, a highly prevalent operon of enterotoxin gene, forms a putative nursery of superantigens in Staphylococcus aureus. The Journal of Immunology. 2001; 166:669-677.

Joshi. 2000. Medicinal plants. Oxford \& IBH publishing Co. Pvt. Ltd. New Delhi. India.

Kim S, Kubec R, Musah RA. Antibacterial and antifungal activity of sulfur-containing compounds from Petiveria alliacea L. Journal of ethnopharmacology. 2006; 104:188-192.

Langran, Dezhao, Xiangyun, Puhua, Zhi, Dianxiang 2009. Fabaceae (Leguminosae), Flora of China.

Liu WC, Kugelman M, Wilson RA, Rao KV. A crystalline saponin with anti-tumor activity from Entada phaseoloides. Phytochemistry. 1972; 11:171-173.

Nzowa L, Teponno R, Tapondjou L, Verotta L, Liao Z, Graham D, Zink M-C, Barboni L. Two new tryptophan derivatives from the seed kernels of Entada rheedei: effects on cell viability and HIV infectivity. Fitoterapia. 2013; 87:37-42.

Okba MM, Soliman FM, EL Deeb KS, Yousif MF. Botanical study, DNA fingerprinting, nutritional values and certain proximates of Entada rheedii Spreng. International Journal of Pharmacy and Pharmaceutical Sciences. 2013; 5(3):31-329.

Okoro IO, Osagie A, Asibor EO. Antioxidant and antimicrobial activities of polyphenols from ethnomedicinal plants of Nigeria. African
Journal of Biotechnology. 2010; 9(20):2989-2993.

Pan H, Lundgren LN. Phenolic extractives from root bark of Picea abies. Phytochemistry. 1995; 39:1423-1428.

Pavithra P, Janani V, Charumathi K, Indumathy R, Potala S, Verma RS. Antibacterial activity of plants used in Indian herbal medicine. International Journal of Green Pharmacy (IJGP). 2010; 4(1):22-28.

Ramakrishna D, Pavan Kumar K, Mukkanti K, Abedulla Khan K. Antiulcer activity of the seeds of Entada phaseoloides. Pharmacologyonline. 2008; 3:93-99.

Repetto M, Llesuy S. Antioxidant properties of natural compounds used in popular medicine for gastric ulcers. Brazilian journal of medical and biological research. 2002; 35:523-534.

Rodríguez E, Sánchez L, Pérez S, Herrera L, Jiménez M, Samper $\mathrm{S}$, Iglesias M. Human tuberculosis due to Mycobacterium bovis and M. caprae in Spain, 2004-2007. The International Journal of Tuberculosis and Lung Disease. 2009; 13:1536-1541.

Smith J. 1960. Chromatographic and Electrophoretic Techniques, William Heinman, London.

Stahl E. 1969. Thin Layer Chromatography, Springer-Verlag,

Berlin.

Sugimoto S, Matsunami K, Otsuka H. Medicinal Plants of Thailand. I Structures of Rheedeiosides AD and cis-Entadamide A. BETA.D-Glucopyranoside from the Seed Kernels of Entada rheedei. Chemical and Pharmaceutical Bulletin. 2011; 59:466-471.

Tapondjou AL, Miyamoto T, Mirjolet J-F, Guilbaud N, LacailleDubois M-A. Pursaethosides A-E, Triterpene Saponins from Entada $p$ ursaetha. Journal of natural products. 2005; 68:1185-1190.

Uddin SN. 2006. Traditional uses of Ethnomedicinal plants of the Chittagong Hill Tracts, Bangladesh National Herbarium.

Vidya S, Krishna V, Manjunatha B, Bharath B, Rajesh K, Manjunatha $\mathrm{H}$, Mankani $\mathrm{K}$. Wound healing phytoconstituents from seed kernel of Entada pursaetha DC. and their molecular docking studies with glycogen synthase kinase 3- $\beta$. Medicinal Chemistry Research. 2012; 21:3195-3203.

Yasuraoka K, Irie Y, Takamura K, Shimomura H, Hashiguchi J, Santos M, Santos A. Jr. Laboratory and field assessment of the molluscicidal activity of gogo (Entada phaseoloides) against the amphibious snail intermediate host of Schistosoma japonicum. The Japanese journal of experimental medicine. 1977; 47:483-487.

Ylipaasto P, Klingel K, Lindberg AM, Otonkoski T, Kandolf R, Hovi T, Roivainen M. Enterovirus infection in human pancreatic islet cells, islet tropism in vivo and receptor involvement in cultured islet beta cells Diabetologia. 2004; 47:225-239.

How to cite this article:

Okba MM, El Awdan SAW, Yousif MF, El Deeb KS, Soliman FM. Entada rheedii seeds thioamides, phenolics, and saponins and its antiulcerogenic and antimicrobial activities. J App Pharm Sci, 2018; 8(05): 101-108. 